\title{
CONDITIONAL ANALYTIC FEYNMAN INTEGRALS ON ABSTRACT WIENER SPACES
}

\author{
DONG MYUNG CHUNG
}

(Communicated by R. Daniel Mauldin)

\begin{abstract}
In this paper we define the concept of a conditional analytic Feynman integral of a function $F$ on an abstract Wiener space $B$ given a function $X$ and then establish the existence of the conditional Feynman integral for all functions in the Fresnel class on $B$. We also use the conditional Feynman integral to provide a fundamental solution to the Schrödinger equation.
\end{abstract}

\section{INTRODUCTION}

Let $H$ be a real separable infinite-dimensional Hilbert space with inner product $\langle\cdot, \cdot\rangle$ and norm $|\cdot|$. Let $\|\cdot\|$ be a measurable norm on $H$ with respect to the Gauss measure $m$ on $H$ (see [12]). It is shown [12] that $\|\cdot\|$ is weaker than $|\cdot|$ on $H$. Let $B$ denote the completion of $H$ with respect to $\|\cdot\|$. Let $i$ denote the natural injection from $H$ into $B$. The adjoint $i^{*}$ of $i$ is one-to-one and maps $B^{*}$ continuously onto a dense subset of $H^{*}$. Thus we have a triple $B^{*} \subset H=H^{*} \subset B$ and $\langle x, y\rangle=(x, y)$ for all $x$ in $H$ and $y$ in $B^{*}$, where $(\cdot, \cdot)$ denotes the natural dual pairing between $B$ and $B^{*}$. By a well-known result of Gross, $m \circ i^{-1}$ has a unique countably-additive extension $\nu$ to the Borel $\sigma$-algebra $\mathscr{B}(B)$ of $B$. The triple $(H, B, \nu)$ is called an abstract Wiener space. For more details, see [7,12]. Let $\mathbf{R}^{n}$ and $\mathbf{C}$ denote an $n$-dimensional Euclidean space and the complex numbers, respectively.

Let $\left(C[0, t], \mathscr{B}(C[0, t]), m_{\mathrm{w}}\right)$ denote Wiener space, that is, $C[0, t]$ denotes the Banach space $\{x(\cdot): x$ is a real-valued continuous function with $x(0)=0\}$ with the supremum norm and $m_{\mathrm{w}}$ denotes the Wiener measure on the Borel $\sigma$-algebra $\mathscr{B}(C[0, t])$ of $C[0, t]$ (see [12]). Let $C^{\prime}[0, t]=\{x \in$ $\left.C[0, t]: x(s)=\int_{0}^{s} f(u) d u, f \in L^{2}[0, t]\right\}$. Then it is a real separable infinitedimerisional Hilbert space with inner product $\left\langle x_{1}, x_{2}\right\rangle=\int_{0}^{t} D x_{1}(\tau) \cdot D x_{2}(\tau) d \tau$, where $D x=d x / d \tau$. As is known, $\left(C[0, t], C^{\prime}[0, t], m_{\mathrm{w}}\right)$ is an example of abstract Wiener spaces (see [12]).

Received by the editors November 17, 1989.

1980 Mathematics Subject Classification (1985 Revision). Primary 28C20.

Key words and phrases. Abstract Wiener space, conditional abstract Wiener integral, Feynman integral, conditional Feynman integral.

Research supported by BSRIP, the Ministry of Education of Korea. 
Let $\left\{e_{j} ; j \geq 1\right\}$ be a complete orthonormal set in $H$ such that $e_{j}$ 's are in $B^{*}$. For each $h \in H$ and $x \in B$, let

$$
(h, x)^{\sim}= \begin{cases}\lim _{n \rightarrow \infty} \sum_{j=1}^{n}\left\langle h, e_{j}\right\rangle\left(e_{j}, x\right), & \text { if the limit exists; } \\ 0, & \text { otherwise } .\end{cases}
$$

Then for each $h(\neq 0)$ in $H,(h, \cdot)^{\sim}$ is a Gaussian random variable on $B$ with mean zero, variance $|h|^{2}$; also and $(h, x)^{\sim}$ is essentially independent of the choice of the complete orthonormal set used in its definition, and further, $(h, \lambda x)^{\sim}=(\lambda h, x)^{\sim}=\lambda(h, x)^{\sim}$ for all $\lambda>0$. It is well known that if $\left\{h_{1}, h_{2}, \ldots, h_{n}\right\}$ is an orthogonal set in $H$, then the random variables $\left(h_{j}, x\right)^{\sim}$ 's are independent and that if $B=C[0, T], H=C^{\prime}[0, T]$, then

$$
(h, x)^{\sim}=\int_{0}^{t} D h(s) \tilde{d} x(s),
$$

where $\int_{0}^{t} D h(s) \tilde{d} x(s)$ is the Paley-Wiener-Zygmund integral of $D h$.

Let $M(H)$ be the class of all $\mathrm{C}$-valued Borel measures on $H$ with bounded variation. Let $\mathscr{F}(H)$ be the class of all functions $f$ on $H$ of the form

$$
f\left(h_{1}\right)=\int_{H} e^{i\left\langle h, h_{1}\right\rangle} d \sigma(h)
$$

for some $\sigma \in M(H) . \mathscr{F}(H)$ is the Fresnel class of Albeverio and Høegh-Krohn [1]. It is known $[10,11]$ that each function of the form (1.1) can be extended to $B$ uniquely by

$$
F(x)=\int_{H} e^{i(h, x)^{\sim}} d \sigma(h) .
$$

Given two $\mathrm{C}$-valued measurable functions $F$ and $G$ on $B, F$ is said to be equal to $G$ s-almost surely (s-a.s.) if for each $\alpha>0, \nu\{x \in B: F(\alpha x) \neq$ $G(\alpha x)\}=0$ (for more detail, see [3]). For a measurable function $F$ on $B$, let [F] denote the equivalence class of functionals which are equal to $F$ s-a.s. The class of equivalence classes defined by

$$
\mathscr{F}(B)=\left\{[F]: F(x)=\int_{H} e^{i(h, x)^{\sim}} d \sigma(h), \sigma \in M(H)\right\}
$$

is called the Fresnel class of functions on $B$. It is known [10] that $\mathscr{F}(B)$ forms a Banach algebra over the complex field and that $\mathscr{F}(H)$ and $\mathscr{F}(B)$ are isometrically isomorphic (see $[8,10]$ ). As is customary, we will identify a function with its $s$-equivalence class and think of $\mathscr{F}(B)$ as a class of functions on $B$ rather than as a class of equivalence classes.

Let $F$ be a $\mathbf{C}$-valued measurable function on $B$ such that the integral

$$
J(\lambda)=\int_{B} F\left(\lambda^{-1 / 2} x\right) d \nu(x)
$$

exists as a finite number for all $\lambda>0$. If there exists a function $J^{*}(\lambda)$, analytic in $\lambda$ on $\mathbf{C}^{+} \equiv\{\lambda \in \mathbf{C}: \operatorname{Re} \lambda>0\}$ such that $J^{*}(\lambda)=J(\lambda)$ for all $\lambda>0$, then 
$J^{*}(\lambda)$ is defined to be the analytic Wiener integral of $F$ over $B$ with parameter $\lambda$, and for $\lambda \in \mathbf{C}^{+}$we write

$$
E^{\mathrm{anw}_{\lambda}}(F)=J^{*}(\lambda)
$$

Let $q \neq 0$ be a real number and let $F$ be a $\mathbf{C}$-valued measurable function such that $E^{\text {anw }_{\lambda}}(F)$ exists for all $\lambda \in \mathbf{C}^{+}$. If the following limit exists, we call it the analytic Feynman integral of $F$ over $B$ with parameter $q$ and we write

$$
E^{\operatorname{anf}_{q}}(F)=\lim _{\lambda \rightarrow-i q} E^{\mathrm{anw}_{i}}(F),
$$

where $\lambda$ approaches $-i q$ through $\mathbf{C}^{+}$.

It is shown in [10, Theorem 3.1] that for $F$ given by (1.2)

$$
E^{\mathrm{anw}_{\lambda}}(F)=\int_{H} \exp \left\{-\frac{1}{2 \lambda}|h|^{2}\right\} d \sigma(h), \quad \lambda \in \mathbf{C}^{+}
$$

and

$$
E^{\operatorname{anf}_{q}}(F)=\int_{H} \exp \left\{-\frac{i}{2 q}|h|^{2}\right\} d \sigma(h)
$$

for each real $c_{i}^{\prime} \neq 0$.

Let $X$ be an $\mathbf{R}^{n}$-valued measurable function and $Y$ a $\mathbf{C}$-valued integrable function on $(B, \mathscr{B}(B), \nu)$. Let $\sigma(X)$ denote the $\sigma$-algebra generated by $X$. Then by the definition of conditional expectation, the conditional expectation of $Y$ given $\sigma(X)$, written $E(Y \mid X)$, is any $\mathbf{R}^{n}$-valued $\sigma(X)$-measurable function on $B$ such that

$$
\int_{E} Y d \nu=\int_{E} E(Y \mid X) d \nu \quad \text { for } E \in \sigma(X) .
$$

It is well known that there exists a Borel measurable and $P_{X}$-integrable function $\psi$ on $\left(\mathbf{R}^{n}, \mathscr{B}\left(\mathbf{R}^{n}\right), P_{X}\right)$ such that $E(Y \mid X)=\psi \circ X$, where $\mathscr{B}\left(\mathbf{R}^{n}\right)$ denotes the Borel $\sigma$-algebra of $\mathbf{R}^{n}$ and $P_{X}$ is the probability distribution of $X$ defined by $P_{X}(A)=\nu\left(X^{-1}(A)\right)$ for $A \in \mathscr{B}\left(\mathbf{R}^{n}\right)$. The function $\psi(\vec{\xi}), \vec{\xi} \in \mathbf{R}^{n}$ is unique up to Borel null sets in $\mathbf{R}^{n}$. Following Yeh [13] the function $\psi(\vec{\xi})$, written $E(Y \mid X)(\vec{\xi})$, is called the conditional abstract Wiener integral of $Y$ given $X$ (see [4]).

In this paper we define the concept of a conditional analytic Feynman integral of a function $F$ on $B$ given an $\mathbf{R}^{n}$-valued function, and then for a certain choice of $X$, we establish the existence of the conditional analytic Feynman integral for all functions $F$ in the Banach algebra $\mathscr{F}(B)$. We also use the conditional analytic Feynman integral over $B$ to provide a fundamental solution to the Schrödinger equation.

An attempt to provide via Feynman integral a fundamental solution to the Schrödinger equation was undertaken by Gelfand and Yaglom in J. Math. Phys. 1 (1960), 48-69. Unfortunately their work contains (on p. 58) a rather major error as pointed out by Cameron in J. Math. Phys. 39 (1960), 126-141. The author thanks the referee for calling his attention to the Gelfand-Yaglom paper. 
We have studied here only the conditional Feynman integrals for the Fresnel class of functions on $B$. The conditional Feynman integral of functions for unbounded potentials will appear in a subsequent paper.

\section{Conditional analytic Feynman integrals on $B$}

We begin with the definition of the conditional analytic Feynman integral of a function $F$ on $B$ given a function $X$ (see $[5,6])$.

Definition 1. Let $X$ be an $\mathbf{R}^{n}$-valued measurable function on $B$ and let $F$ be a $C$-valued measurable function on $B$ such that the integral

$$
\int_{B} F\left(\lambda^{-1 / 2} x\right) d \nu(x)
$$

exists as a finite number for all $\lambda>0$. For $\lambda>0$ let

$$
J_{\lambda}(\vec{\eta})=E\left(F\left(\lambda^{-1 / 2} \cdot\right) \mid X\left(\lambda^{-1 / 2} \cdot\right)\right)(\vec{\eta})
$$

denote the conditional abstract Wiener integral of $F\left(\lambda^{-1 / 2} \cdot\right)$ given $X\left(\lambda^{-1 / 2} \cdot\right)$. If for a.e. $\vec{\eta} \in \mathbf{R}^{n}$, there exists a function $J_{\lambda}^{*}(\vec{\eta})$, analytic in $\lambda$ on $\mathbf{C}^{+}$such that $J_{\lambda}^{*}(\vec{\eta})=J_{\lambda}(\vec{\eta})$ for all $\lambda>0$, then $J_{\lambda}^{*}$ is defined to be the conditional analytic Wiener integral of $F$ over $B$ given $X$ with parameter $\lambda$ and for $\lambda \in \mathbf{C}^{+}$we write

$$
E^{\mathrm{anw}_{\lambda}}(F \mid X)(\vec{\eta})=J_{\lambda}^{*}(\vec{\eta}) .
$$

If for fixed real $q \neq 0$, the limit

$$
\lim _{\lambda \rightarrow-i q} E^{\mathrm{anw}}(F \mid X)(\vec{\eta})
$$

exists for a.e. $\vec{\eta} \in \mathbf{R}^{n}$ where $\lambda$ approaches $-i q$ through $\mathbf{C}^{+}$, then we will denote the value of this limit by $E^{\text {anf }_{q}}(F \mid X)$ and call it the conditional analytic Feynman integral of $F$ over $B$ given $X$ with parameter $q$.

Remark 1. The notation $E^{\mathrm{anw}_{\lambda}}(F \mid X)$ does not mean "conditional expectation with respect to a probability measure" but rather an extension of such a conditional expectation.

We now consider conditioning functions on $B$ of the form

$$
X(x)=\left(\left(g_{1}, x\right)^{\sim},\left(g_{2}, x\right)^{\sim}, \ldots,\left(g_{n}, x\right)^{\sim}\right),
$$

where $\left\{g_{1}, g_{2}, \ldots, g_{n}\right\}$ is an orthonormal subset of $H$, and then establish the existence of the conditional Feynman integral for all $F$ in $\mathscr{F}(B)$ given a conditioning function $X$ of the form (2.1). We note that if $h$ is in $H$, then

$$
X(h)=\left(\left\langle g_{1}, h\right\rangle,\left\langle g_{2}, h\right\rangle, \ldots,\left\langle g_{n}, h\right\rangle\right) .
$$

Theorem 1. Let $F \in \mathscr{F}(B)$ be given by (1.2), and let $X(x)$ be as in (2.1). Then for all $\lambda \in \mathbf{C}^{+}$, the conditional analytic Wiener integral over $B, E^{\text {anw }_{\lambda}}(F \mid X)$ exists and for all $\vec{\eta} \in \mathbf{R}^{n}$ is given by the formula

$$
E^{\mathrm{anw}_{\lambda}}(F \mid X)(\vec{\eta})=\int_{H} \exp \left\{-\frac{1}{2 \lambda}\left(|h|^{2}-|X(h)|^{2}\right)+i\langle X(h), \vec{\eta}\rangle\right\} d \sigma(h),
$$


where $X(h)$ is as in (2.2). Furthermore the conditional analytic Feynman integral $E^{\operatorname{anf}_{q}}(F \mid X)$ exists for all $q \neq 0$ and for all $\vec{\eta} \in \mathbf{R}^{n}$ is given by the formula

$$
E^{\operatorname{anf}_{q}}(F \mid X)(\vec{\eta})=\int_{H} \exp \left\{-\frac{i}{2 q}\left(|h|^{2}-|X(h)|^{2}\right)+i\langle X(h), \vec{\eta}\rangle\right\} d \sigma(h) .
$$

Proof. Let $h \in H$ be fixed. Then $h$ can be written as

$$
h=\sum_{j=1}^{n}\left\langle g_{j}, h\right\rangle g_{j}+p, \quad p \in\left[g_{1}, g_{2}, \ldots, g_{n}\right]^{\perp},
$$

where $[A]^{\perp}$ denotes the orthogonal complement of the subspace of $H$ spanned by $A$. Thus we have

$$
\exp \left\{i(h, x)^{\sim}\right\}=\exp \left\{i \sum_{j=1}^{n}\left\langle g_{j}, h\right\rangle\left(g_{j}, x\right)^{\sim}\right\} \exp \left\{i(p, x)^{\sim}\right\} .
$$

Therefore we have

$$
\begin{aligned}
E\left(F\left(\lambda^{-1 / 2} \cdot\right) \mid X\left(\lambda^{-1 / 2} \cdot\right)\right)= & E\left(\int_{H} \exp \left\{i\left(h, \lambda^{-1 / 2} x\right)^{\sim}\right\} d \sigma(h) \mid X\left(\lambda^{-1 / 2} \cdot\right)\right) \\
= & \int_{H} E\left(\exp \left\{i\left(h, \lambda^{-1 / 2} x\right)^{\sim}\right\} \mid X\left(\lambda^{-1 / 2} \cdot\right)\right) d \sigma(h) \\
= & \int_{H} \exp \left\{i \sum_{j=1}^{n}\left\langle g_{j}, h\right\rangle\left(g_{j}, \lambda^{-1 / 2} x\right)^{\sim}\right\} \\
& \times E\left(\exp \left\{i\left(p, \lambda^{-1 / 2} x\right)^{\sim}\right\} \mid X\left(\lambda^{-1 / 2} \cdot\right)\right) d \sigma(h) .
\end{aligned}
$$

But $\left(g_{j}, x\right)^{\sim}$ 's and $(p, x)^{\sim}$ are independent, so that

$$
E\left(\exp \left\{i \lambda^{-1 / 2}(p, x)^{\sim}\right\} \mid X\left(\lambda^{-1 / 2} \cdot\right)\right)=E\left(\exp \left\{i \lambda^{-1 / 2}(p, x)^{\sim}\right\}\right) .
$$

Since $E\left(\exp \left\{i \lambda^{-1 / 2}(p, x)^{\sim}\right\}\right)=\exp \left\{-(1 / 2 \lambda)|p|^{2}\right\}$, it follows that

$$
\begin{aligned}
& E\left[F\left(\lambda^{-1 / 2} \cdot\right) \mid X\left(\lambda^{-1 / 2} \cdot\right)\right](\vec{\eta}) \\
& \quad=\int_{H} \exp \{i\langle X(h), \vec{\eta}\rangle\} \exp \left\{-\frac{1}{2 \lambda}\left(|h|^{2}-|X(h)|^{2}\right)\right\} d \sigma(h) .
\end{aligned}
$$

But $|h|^{2}-|X(h)|^{2} \geq 0$ for all $h \in H$. Hence since $\sigma \in M(H)$, the right-hand side of (2.5) is an analytic function of $\lambda$ throughout $\mathbf{C}^{+}$and is continuous of $\lambda$ for $\operatorname{Re} \lambda \geq 0, \lambda \neq 0$. Hence we establish the equations (2.3) and (2.4) as desired.

Example. Let $B=C[0, t]$ and $H=C^{\prime}[0, t]$. Let us fix a partition $\left\{0=t_{0}<\right.$ $\left.t_{1}<\cdots<t_{n}=t\right\}$ of $[0, t]$ and let $g_{j} \in C^{\prime}[0, t]$ be defined by

$$
g_{j}(s)=\left(t_{j}-t_{j-1}\right)^{-1 / 2} \int_{0}^{s} 1_{\left[t_{j-1}, t_{j}\right)}(u) d u, \quad j=1, \ldots, n .
$$


Then $\left\{g_{1}, g_{2}, \ldots, g_{n}\right\}$ is an orthonormal set in $C^{\prime}[0, t]$ and

$$
|X(h)|^{2}=\sum_{j=1}^{n}\left\langle g_{j}, h\right\rangle^{2}=\sum_{j=1}^{n} \frac{1}{t_{j}-t_{j-1}} \int_{t_{j-1}}^{t_{j}} D h(s) d s \quad \text { for all } h \in C^{\prime}[0, t] .
$$

We note that for $x \in C[0, t],\left(\left(x\left(t_{1}\right), x\left(t_{2}\right), \ldots, x\left(t_{n}\right)\right)=\left(\eta_{1}, \eta_{2}, \ldots, \eta_{n}\right)\right.$ if and only if $\left(g_{j}, x\right)^{\sim}=\left(t_{j}-t_{j-1}\right)^{-1 / 2}\left(\eta_{j}-\eta_{j-1}\right)$ for all $j=1,2, \ldots, n$, where $\eta_{0}=0$.

Let $\sigma$ be a C-valued Borel measure on $L^{2}[0, t]$ with bounded variation. If $F$ is given by

$$
F(x)=\int_{L^{2}[0, t]} \exp \left\{i \int_{0}^{t} v(s) \tilde{d} x(s)\right\} d \sigma(v) \quad \text { s-a.s. } x
$$

(see [2]), then using Theorem 1, we obtain

$$
\begin{aligned}
E^{\mathrm{anf}_{a}}\left[F \mid X=\left(x\left(t_{1}\right), \ldots, x\left(t_{n}\right)\right)\right]\left(\left(\eta_{1}, \ldots, \eta_{n}\right)\right) & \\
=\int_{L^{2}[0, t]} \exp \left\{-\frac{i}{2 q}\left[\int_{0}^{t}|v(s)|^{2} d s\right.\right. & \left.-\sum_{j=1}^{n} \frac{1}{t_{j}-t_{j-1}}\left(\int_{t_{j-1}}^{t_{j}} v(s) d s\right)^{2}\right] \\
& \left.+i \sum_{j=1}^{n} \frac{\eta_{j}-\eta_{j-1}}{t_{j}-t_{j-1}} \int_{t_{j-1}}^{t_{j}} v(s) d s\right\} d \sigma(v) .
\end{aligned}
$$

In our next theorem, we need the following summation procedure (see [9], p. 340$)$ :

$$
\int_{\mathbf{R}^{n}} f(\vec{\eta}) d \vec{\eta}=\lim _{A \rightarrow \infty} \int_{\mathbf{R}^{n}} f(\vec{\eta}) \exp \left\{-\frac{|\vec{\eta}|^{2}}{2 A}\right\} d \vec{\eta}
$$

whenever the expression on the right exists. Of course if $f \in L^{1}\left(\mathbf{R}^{n}\right)$, it is clear using the dominated convergence theorem that

$$
\int_{\mathbf{R}^{n}} f(\vec{\eta}) d \vec{\eta}=\int_{\mathbf{R}^{n}} f(\vec{\eta}) d \vec{\eta} .
$$

Theorem 2. Let $F$ and $X$ be as in Theorem 1. Then for all $\lambda \in \mathbf{C}^{+}$

$$
\int_{\mathbf{R}^{n}}\left(\frac{\lambda}{2 \pi}\right)^{n / 2} \exp \left\{-\frac{\lambda}{2}|\vec{\eta}|^{2}\right\} E^{\mathrm{anw}_{\lambda}}(F \mid X)(\vec{\eta}) d \vec{\eta}=E^{\mathrm{anw}_{\lambda}}(F)
$$

and for all real $q \neq 0$,

$$
\bar{\int}_{\mathbf{R}^{n}}\left(\frac{q}{2 \pi i}\right)^{n / 2} \exp \left\{\frac{i q}{2}|\vec{\eta}|^{2}\right\} E^{\operatorname{anf}_{q}}(F \mid X)(\vec{\eta}) d \vec{\eta}=E^{\operatorname{anf}_{q}}(F) .
$$


Proof. We will establish equation (2.10); the proof of (2.9) is similar, but easier since the summation procedure is not needed. Let $q \neq 0$ be given. Then using (2.8), (2.4), the Fubini Theorem, and (1.3) we obtain

$$
\begin{aligned}
\int_{\mathbf{R}^{n}} & \left.\frac{q}{2 \pi i}\right)^{n / 2} \exp \left\{\frac{i q}{2}|\vec{\eta}|^{2}\right\} E^{\operatorname{anf}_{q}(F \mid X)(\vec{\eta}) d \vec{\eta}} \\
= & \lim _{A \rightarrow \infty} \int_{\mathbf{R}^{n}}\left(\frac{q}{2 \pi i}\right)^{n / 2} \exp \left\{\frac{1}{2}\left(i q-\frac{1}{A}\right)|\vec{\eta}|^{2}\right\} \\
& \times \int_{H} \exp \left\{-\frac{i}{2 q}\left(|h|^{2}-|X(h)|^{2}\right)+i\langle X(h), \vec{\eta}\rangle\right\} d \sigma(h) d \vec{\eta} \\
= & \lim _{A \rightarrow \infty} \int_{H} \exp \left\{-\frac{i}{2 q}\left(|h|^{2}-|X(h)|^{2}\right)\right\}\left(\frac{q}{2 \pi i}\right)^{n / 2} \\
& \times \int_{\mathbf{R}^{n}} \exp \left\{-\frac{1}{2}\left(\frac{1-A i q}{A}\right)|\vec{\eta}|^{2}+i\langle X(h), \vec{\eta}\rangle\right\} d \vec{\eta} d \sigma(h) \\
= & \lim _{A \rightarrow \infty} \int_{H} \exp \left\{-\frac{i}{2 q}\left(|h|^{2}-|X(h)|^{2}\right)\right\}\left(\frac{q}{2 \pi i}\right)^{n / 2} \\
& \times\left(\frac{2 \pi A}{1-A i q}\right)^{n / 2} \exp \left\{-\frac{A|X(h)|^{2}}{2(1-A i q)}\right\} d \sigma(h) \\
= & \int_{H} \exp \left\{-\frac{i}{2 q}\left(|h|^{2}-|X(h)|^{2}\right)\right\} \exp \left\{-\frac{i}{2 q}|X(h)|^{2}\right\} d \sigma(h) \\
= & \int_{H} \exp \left\{-\frac{i}{2 q}|h|^{2}\right\} d \sigma(h)=E^{\operatorname{anf}_{q}}(F) .
\end{aligned}
$$

\section{AN APPLication}

In this section we use the conditional analytic Feynman integral to provide a fundamental solution to the Schrödinger equation.

Theorem 3. Let $F$ and $X$ be as in Theorem 1. Let $\psi$ be given by

$$
\psi(\vec{\eta})=\int_{\mathbf{R}^{n}} \exp \{i\langle\vec{y}, \vec{\eta}\rangle\} d \phi(\vec{y}),
$$

where $\phi$ is a complex Borel measure on $\mathbf{R}^{n}$ with bounded variation. For $(t, \vec{\eta}) \in$ $(0, \infty) \times \mathbf{R}^{n}$, let

$$
G(x) \equiv G_{t, \vec{\eta}}(x)=F(x) \psi(X(x)+\vec{\eta}) .
$$

Then for all $q \neq 0$ we have that

$$
\begin{aligned}
\Gamma(t, \vec{\eta}, q) \equiv & E^{\mathrm{anf}_{q}}(G) \\
= & \int_{H}\left[\exp \left\{-\frac{i}{2 q}\left(|h|^{2}-|X(h)|^{2}\right)\right\}\right. \\
& \left.\times \int_{\mathbf{R}^{n}} \exp \left\{i\langle\vec{\eta}, \vec{y}\rangle-\frac{1}{2 q}|X(h)+\vec{y}|^{2}\right\} d \phi(\vec{y})\right] d \sigma(h) .
\end{aligned}
$$


In addition we have the alternative expression

$$
E^{\operatorname{anf}_{q}}(G)=\int_{\mathbf{R}^{n}} E^{\operatorname{anf}_{q}}(F \mid X)(\vec{\xi}-\vec{\eta})\left(\frac{q}{2 \pi i}\right)^{n / 2} \exp \left\{\frac{i q|\vec{\xi}-\vec{\eta}|^{2}}{2}\right\} \psi(\vec{\xi}) d \vec{\xi}
$$

where $E^{\operatorname{anf}_{q}}(F \mid X)(\cdot)$ is given by formula (2.4).

Proof. Since $P_{X(\cdot)+\vec{\eta}}(d \vec{\xi})=(2 \pi)^{-n / 2} \exp \left\{-|\vec{\xi}-\vec{\eta}|^{2} / 2\right\} d \vec{\xi}$, by [13, Lemma 1] it follows that

$$
\begin{aligned}
J(\lambda) & =\int_{B} G\left(\lambda^{-1 / 2} x\right) d \nu(x) \\
& =\int_{B} F\left(\lambda^{-1 / 2} x\right) \psi\left(X\left(\lambda^{-1 / 2} x\right)+\vec{\eta}\right) d \nu(x) \\
& =\int_{\mathbf{R}^{n}} E\left(F\left(\lambda^{-1 / 2} \cdot\right) \mid X\left(\lambda^{-1 / 2} \cdot\right)+\vec{\eta}\right)(\vec{\xi}) \psi(\vec{\xi})\left(\frac{\lambda}{2 \pi}\right)^{n / 2} \exp \left\{-\frac{\lambda|\vec{\xi}-\vec{\eta}|^{2}}{2}\right\} d \vec{\xi} \\
& =\int_{\mathbf{R}^{n}} E\left(F\left(\lambda^{-1 / 2} \cdot\right) \mid X\left(\lambda^{-1 / 2} \cdot\right)\right)(\vec{\xi}-\vec{\eta}) \psi(\vec{\xi})\left(\frac{\lambda}{2 \pi}\right)^{n / 2} \exp \left\{-\frac{\lambda|\vec{\xi}-\vec{\eta}|^{2}}{2}\right\} d \vec{\xi}
\end{aligned}
$$

for all $\lambda>0$. Then using Theorem 1 and Morera's Theorem, we obtain that

$$
E^{\mathrm{anw}_{\lambda}}(G)=\int_{\mathbf{R}^{n}} E^{\mathrm{anw}_{\lambda}}(F \mid X)(\vec{\xi}-\vec{\eta})\left(\frac{\lambda}{2 \pi}\right)^{n / 2} \exp \left\{-\frac{\lambda|\vec{\xi}-\vec{\eta}|^{2}}{2}\right\} \psi(\vec{\xi}) d \vec{\xi}
$$

for all $\lambda \in \mathbf{C}^{+}$. Next we substitute for $E^{\text {anw }_{\lambda}}(F \mid X)(\vec{\xi}-\vec{\eta})$ and $\psi(\vec{\xi})$ in (3.4) using (2.3) and (3.1), use the Fubini Theorem and then carry out the integration with respect to $\vec{\xi}$ and obtain the formula

$$
\begin{aligned}
E^{\mathrm{anw}_{\lambda}}(G)=\int_{H}[ & \exp \left\{-\frac{1}{2 \lambda}\left(|h|^{2}-|X(h)|^{2}\right)\right\} \\
& \left.\times \int_{\mathbf{R}^{n}} \exp \left\{i\langle\vec{\eta}, \vec{y}\rangle-\frac{1}{2 \lambda}|X(h)+\vec{y}|^{2}\right\} d \phi(\vec{y})\right] d \sigma(h)
\end{aligned}
$$

for all $\lambda \in \mathbf{C}^{+}$. Next we note that the right-hand side of (3.5) is continuous in $\lambda$ for $\operatorname{Re} \lambda \geq 0, \lambda \neq 0$ and hence $E^{\operatorname{anf}_{q}}(G)$ exists and is given by (3.2).

To obtain the alternative expression (3.3), we use $(2.8),(2.4)$, the domi- 
nated convergence theorem, and (3.2):

$$
\begin{aligned}
& \bar{\int}_{\mathbf{R}^{n}} E^{\operatorname{anf}_{q}}(F \mid X)(\vec{\xi}-\vec{\eta})\left(\frac{q}{2 \pi i}\right)^{n / 2} \exp \left\{\frac{i q}{2}|\vec{\xi}-\vec{\eta}|^{2}\right\} \psi(\vec{\xi}) d \vec{\xi} \\
& =\lim _{A \rightarrow \infty} \int_{\mathbf{R}^{n}} E^{\operatorname{anf}_{q}}(F \mid X)(\vec{\xi}-\vec{\eta})\left(\frac{q}{2 \pi i}\right)^{n / 2} \exp \left\{\frac{i q}{2}|\vec{\xi}-\vec{\eta}|^{2}-\frac{1}{2 A}|\vec{\xi}|^{2}\right\} \psi(\vec{\xi}) d \vec{\xi} \\
& =\lim _{A \rightarrow \infty} \int_{\mathbf{R}^{n}}\left[\int_{H} \exp \left\{-\frac{i}{2 q}\left(|h|^{2}-|X(h)|^{2}\right)+i\langle X(h), \vec{\xi}-\vec{\eta}\rangle\right\} d \sigma(h)\right. \\
& \left.\times\left(\frac{q}{2 \pi i}\right)^{n / 2} \exp \left\{\frac{i q}{2}|\vec{\xi}-\vec{\eta}|^{2}-\frac{1}{2 A}|\vec{\xi}|^{2}\right\} \int_{\mathbf{R}^{n}} \exp \{i\langle\vec{\xi}, \vec{y}\rangle\} d \phi(\vec{y})\right] d \vec{\xi} \\
& =\lim _{A \rightarrow \infty} \int_{H}\left[\int_{\mathbf{R}^{n}} \exp \left\{-\frac{i}{2 q}\left(|h|^{2}-|X(h)|^{2}\right)-i\langle X(h), \vec{\eta}\rangle\right\}\left(\frac{q}{2 \pi i}\right)^{n / 2}\right. \\
& \left.\left.\times \int_{\mathbf{R}^{n}} \exp \{i\langle X(h), \vec{\xi}\rangle\}+\frac{i q}{2}|\vec{\xi}-\vec{\eta}|^{2}-\frac{1}{2 A}|\vec{\xi}|^{2}+i\langle\vec{\xi}, \vec{y}\rangle\right\} d \vec{\xi} d \phi(\vec{y})\right] d \sigma(h) \\
& =\lim _{A \rightarrow \infty} \int_{H}\left[\int_{\mathbf{R}^{n}} \exp \left\{-\frac{i}{2 q}\left(|h|^{2}-|X(h)|^{2}\right)-i\langle X(h), \vec{\eta}\rangle+\frac{i q}{2}|\vec{\eta}|^{2}\right\}\left(\frac{q}{2 \pi i}\right)^{n / 2}\right. \\
& \left.\times\left[\int_{\mathbf{R}^{n}} \exp \left\{-\frac{(1-A i q)}{2 A}|\vec{\xi}|^{2}+i\langle X(h)-q \vec{\eta}+\vec{y}, \vec{\xi}\rangle\right\} d \vec{\xi}\right] d \phi(\vec{y})\right] d \sigma(h) \\
& =\lim _{A \rightarrow \infty} \int_{H}\left[\exp \left\{-\frac{i}{2 q}\left(|h|^{2}-|X(h)|^{2}\right)-i\langle X(h), \vec{\eta}\rangle+\frac{i q}{2}|\vec{\eta}|^{2}\right\}\right. \\
& \left.\times\left(\frac{q}{2 \pi i}\right)^{n / 2}\left(\frac{2 \pi A}{1-A i q}\right)^{n / 2} \int_{\mathbf{R}^{n}} \exp \left\{-\frac{A|X(h)-q \vec{\eta}+\vec{y}|^{2}}{2(1-A i q)}\right\} d \phi(\vec{y})\right] d \sigma(h) \\
& =\int_{H}\left[\exp \left\{-\frac{i}{2 q}\left(|h|^{2}-|x(h)|^{2}\right)\right\}\right. \\
& \left.\therefore \int_{\mathbf{R}^{n}} \exp \left\{i\langle\vec{\eta}, \vec{y}\rangle-\frac{i}{2 q}|X(h)+\vec{y}|^{2}\right\} d \phi(\vec{y})\right] d \sigma(h)=E^{\operatorname{anf}_{q}}(G) .
\end{aligned}
$$

Remark 2. Let $H(t, \vec{\eta}, q)=E^{\operatorname{anf}_{q}}(F \mid X)(-\vec{\eta})\left(\frac{q}{2 \pi i}\right)^{n / 2} \exp \left\{\frac{i q}{2}|\vec{\eta}|^{2}\right\}$. Then we note that equation (3.3) may be written as $\Gamma(t, \vec{\eta}, q)=H(t,(\cdot), q) * \psi(\vec{\eta})$ where * denotes convolution. If we specialize Theorem 3 to Wiener space $C[0, t]$, then we see from the example from $\S 1$ that

$$
\begin{aligned}
H(t, \vec{\eta}, q)= & \prod_{j=1}^{n}\left(\frac{q}{2 \pi i\left(t_{j}-t_{j-1}\right)}\right)^{1 / 2} \\
& \times \exp \left\{\frac{i q}{2} \sum_{j=1}^{n} \frac{\left(\eta_{j}-\eta_{j-1}\right)^{2}}{t_{j}-t_{j-1}}\right\} E^{\operatorname{anf}_{q}}(F \mid X)(-\vec{\eta}),
\end{aligned}
$$

where $E^{\operatorname{anf}_{q}}(F \mid X)(\cdot)$ is as in (2.7). By the results of [1, Theorem 3.1], and [8], (3.3) shows that $H(t, \vec{\eta}, q)$ with $q=m / \hbar$ is a fundamental solution to the Schrödinger equation:

$$
i \hbar \frac{\partial \Gamma}{\partial t}=-\frac{\hbar^{2}}{2 m} \Delta \Gamma+V(\vec{\eta}) \Gamma, \quad \Gamma(0, \vec{\eta})=\psi(\vec{\eta}),
$$


where $\Delta$ is the Laplacian on $\mathbf{R}^{n}, \hbar=h / 2 \pi, h$ is Planck's constant, $V$ is a real-valued function of the form (3.1), and $\psi$ is as in (3.1) with $\psi \in L^{2}\left(\mathbf{R}^{n}\right)$.

\section{REFERENCES}

1. A. Albeverio and R. Høegh-Krohn, Mathematical theory of Feynman path integrals, Lecture Notes in Math., vol. 523, Springer-Verlag, Berlin, 1976.

2. R. H. Cameron and D. A. Storvick, Some Banach algebras of analytic Feynman integrable functionals, Analytic Functions, Kozubnik 1979, Lecture Notes in Math., vol. 798, SpringerVerlag, Berlin, 1980, pp. 18-67.

3. D. M. Chung, Scale-invariant measurability in abstract Wiener spaces, Pacific J. Math. 130 (1987), 27-40.

4. D. M. Chung and S. J. Kang, Evaluation formulas for conditional abstract Wiener integrals, Stochastic Analysis and Applications 7 (1989), 125-144.

5. D. M. Chung and D. Skoug, Conditional analytic Feynman integrals and a related Schrödinger integral equation, SIAM on Mathematical Analysis, vol. 20, 1989, pp. 950-965.

6. D. M. Chung, C. Park, and D. Skoug, Operator-valued Feynman integral via conditional Feynman integrals, Pacific J. Math. (to appear).

7. L. Gross, Abstract Wiener space, Proc. 5th Berkeley Sympos. Math. Stat. Prob. 2 (1965), 31-42.

8. G. W. Johnson, The equivalence of two approaches to the Feynman integral, J. Math. Phys., 23 (1982), 2090-2096.

9. G. W. Johnson and D. L. Skoug, Notes on the Feynman integral, III: The Schrödinger equation, Pacific J. Math. 105 (1983), 321-358.

10. G. Kallianpur and C. Bromley, Generalized Feynman integrals using analytic continuation in several complex variables, Stochastic Analysis (M. Pinsky, ed.), Marcel-Dekker, 1984.

11. G. Kallianpur, D. Kannan, and R. L. Karandikar, Analytic and sequential Feynman integrals on abstract Wiener and Hilbert spaces and a Cameron-Martin formula, Ann. Inst. H. Poincaré 21 (1985), 323-361.

12. H. H. Kuo, Gaussian measures in Banach spaces, Lecture Notes in Math., vol. 463, SpringerVerlag, Berlin, 1975.

13. J. Yeh, Inversion of conditional Wiener integrals, Pacific J. Math. 59 (1975), 623-638.

Department of Mathematics, Sogang University, Seoul 121-742, Korea 